\title{
Effects of Prior Contest Experience and Contest Outcome on Female Reproductive Decisions and Offspring Fitness
}

\author{
Natalie Pilakouta, ${ }^{1, \star}$ Cerian Halford, ${ }^{1}$ Rita Rácz, ${ }^{2}$ and Per T. Smiseth ${ }^{1, \dagger}$ \\ 1. Institute of Evolutionary Biology, University of Edinburgh, Edinburgh EH9 3JT, United Kingdom; 2. Department of Evolutionary \\ Zoology and Human Biology, University of Debrecen, Debrecen H-4032, Hungary; and MTA-DE (Hungarian Academy of Sciences- \\ University of Debrecen) Lendület Behavioural Ecology Research Group, Debrecen H-4032, Hungary
}

Submitted November 5, 2015; Accepted April 11, 2016; Electronically published June 21, 2016

Dryad data: http://dx.doi.org/10.5061/dryad.fq22f.

\begin{abstract}
Winning or losing a prior contest can influence the outcome of future contests, but it might also alter subsequent reproductive decisions. For example, losers may increase their investment in the current breeding attempt if losing a contest indicates limited prospects for future breeding. Using the burying beetle Nicrophorus vespilloides, we tested whether females adjust their prehatching and posthatching reproductive effort after winning or losing a contest with a same-sex conspecific. Burying beetles breed on carcasses of small vertebrates for which there is fierce intrasexual competition. We found no evidence that winning or losing a contest influenced reproductive investment decisions in this species. Instead, we show that a female's prior contest experience (regardless of its outcome) influenced the amount of posthatching care provided, with downstream consequences for the female's reproductive output; both winners and losers spent more time provisioning food to their offspring and produced larger broods than females with no contest experience. We discuss the wider implications of our findings and present a conceptual model linking contestmediated adjustments in parental investment to population-level processes. We propose that the frequency of intraspecific contests could both influence and be influenced by population dynamics in species where contest experience influences the size and/or number of offspring produced.
\end{abstract}

Keywords: fighting contest, Nicrophorus vespilloides, parental care, population density, reproductive investment, winner-loser effects.

\section{Introduction}

Animals commonly fight over mates, territories, food, and other resources. Winner-loser effects occur when an individual's prior experience with a fighting contest influences the outcome of its subsequent contests. Previous work in a wide range of taxa has shown that the winner of a contest is more likely to win a future fight, whereas the loser is

* Corresponding author; e-mail: n.pilakouta@gmail.com.

† ORCIDs: Pilakouta, http://orcid.org/0000-0001-8503-520X; Rácz, http:// orcid.org/0000-0002-3049-2048; Smiseth, http://orcid.org/0000-0001-6896 -1332 .

Am. Nat. 2016. Vol. 188, pp. 319-328. (C) 2016 by The University of Chicago. 0003-0147/2016/18803-56635\$15.00. All rights reserved. DOI: $10.1086 / 687392$ more likely to lose again (Chase et al. 1994; Safryn and Scott 2000; Hsu et al. 2006; Rutte et al. 2006; Fawcett and Johnstone 2010; Kasumovic et al. 2010). Two recent studies have challenged this idea by showing that both winners and losers have increased fighting success in future contests (Benelli et al. 2015a, 2015b). This suggests that prior experience with a fight, regardless of its outcome, may give individuals an advantage over inexperienced individuals.

Despite an extensive literature on winner-loser effects, little is known about the wider implications of winning or losing a fight beyond an effect on success in future fights. For example, the outcome of a contest may influence an individual's subsequent reproductive decisions by providing information about its size and condition relative to its competitors (Hsu and Wolf 2001; Walling et al. 2008; Okada et al. 2010). We are aware of only one study that has investigated the effects of contest outcome in the context of reproduction. Okada et al. (2010) compared male flour beetles (Gnatocerus cornutus) that lost or won a prior contest and found that losers transferred more sperm during a subsequent copulation compared to winners. Although this study shows that losing a contest can affect sperm allocation in males, there is no information as to whether contest outcome may also influence decisions over parental effort. If losing indicates limited prospects for success in future breeding attempts, a loser might increase its parental effort in the current breeding attempt, thereby altering both its own fitness and the fitness of its offspring. Alternatively, individuals might base their reproductive investment decisions on whether they have participated in a fighting contest rather than whether they won or lost such a contest. This could occur when encounters with conspecific competitors serve as a cue for the intensity of competition in the population and therefore the likelihood of future breeding opportunities.

Our study addresses this gap in our knowledge using the burying beetle Nicrophorus vespilloides, which breeds on carcasses of small vertebrates. A number of attributes make 
this species particularly well suited for studying how contest outcome and contest experience might affect parental care decisions. First, there is fierce intrasexual competition over the possession of a carcass, which is an ephemeral, highvalue resource (Safryn and Scott 2000). Body size is the strongest determinant of the outcome of these contests, with larger beetles being more successful at both acquiring and defending a carcass (Bartlett and Ashworth 1988). A study on the related Nicrophorus humator reported evidence for winner-loser effects, as the outcome of a prior contest affected the likelihood of success in subsequent contests (Otronen 1990). Second, N. vespilloides females (sometimes assisted by a male) provide elaborate parental care that enhances larval growth and survival (Eggert et al. 1998; Smiseth and Moore 2002; Smiseth et al. 2003; Pilakouta et al. 2015a). Prehatching care includes preparation of the carcass and investment of nutrients in eggs (Rozen et al. 2008; Monteith et al. 2012), while posthatching care includes brood defense, secretion of antimicrobials, and food provisioning (Eggert et al. 1998; Smiseth et al. 2003; Rozen et al. 2008). Last, there is evidence for a trade-off between investment in current and future reproduction in $N$. vespilloides and the related Nicrophorus orbicollis: females that overproduce offspring in the first breeding attempt suffer a reduction in fecundity in future breeding attempts (Creighton et al. 2009; Ward et al. 2009; Billman et al. 2014).

The aim of our study was to investigate whether females adjust their parental effort in the current brood depending on whether they won or lost a prior contest. We focused on female reproductive decisions because females are more involved in care and stay on the carcass longer than males (Fetherston et al. 1994; Eggert et al. 1998; Smiseth and Moore 2002; Rauter and Moore 2004; Smiseth et al. 2005). Furthermore, there is no evidence that the additional presence of a male has a positive effect on larval growth or survival under laboratory conditions (Smiseth et al. 2005). To avoid a possible confounding effect of body size on the reproductive decisions of females (Steiger 2013), we compared medium-sized females with no fighting experience (controls) with medium-sized females that either won or lost a prior contest to small or large females, respectively. Although we hypothesized that the outcome of a prior contest would influence subsequent reproductive decisions, females might also adjust their reproductive investment simply due to their experience with a contest, regardless of its outcome. We thus used an experimental design that would allow us to disentangle the potential effects due to experience with a prior contest and the outcome of that contest (see "Methods").

We first tested whether females adjusted their prehatching investment (egg number and egg size) and posthatching investment (amount of direct care and amount of indirect care) based on contest outcome or contest expe- rience. We then tested whether any adjustments in investment had fitness consequences for the mother by measuring brood size (a measure of reproductive output) and maternal postbreeding longevity (a measure of residual reproductive value). Last, to determine whether contest outcome or contest experience had consequences for offspring fitness, we measured larval begging rate during early development and average larval mass at the dispersal stage, which corresponds to the end of the parental care period. We predicted that losers would increase their investment in current reproduction and consequently suffer higher mortality after breeding. As a result of such an increase in parental investment, we also expected that losers would have more and/or larger offspring at the end of the parental care period.

\section{Methods}

We used virgin beetles from an outbred laboratory population maintained at the University of Edinburgh. The beetles used in this study comprised fourth-, fifth-, and sixthgeneration beetles from lines originally collected in Edinburgh, United Kingdom, and Warmond, the Netherlands. All adult beetles were housed individually in transparent plastic containers $(12 \mathrm{~cm} \times 8 \mathrm{~cm} \times 2 \mathrm{~cm})$ filled with moist soil and kept at $20^{\circ} \mathrm{C}$ and constant light (Mattey and Smiseth 2015a). All nonbreeding adults were fed small pieces of raw organic beef twice a week.

\section{Experimental Design}

Given that the outcome of contests over the possession of a carcass is largely determined by body size (Bartlett and Ashworth 1988) and that larger females provide better care to their offspring (Steiger 2013), it was essential to use an experimental design that would allow us to separate effects due to winning or losing a contest from effects due to body size. To this end, we compared medium-sized females that had different experiences from a prior contest; these medium-sized females competed with either larger or smaller females and thus lost or won the contest, respectively. This design excludes confounding effects of focal female size but not of competitor size, and it does not separate the effects of contest experience from effects of merely encountering a conspecific.

Because adult body size is determined by larval mass at the dispersal stage (Bartlett and Ashworth 1988; Lock et al. 2004), it is possible to generate different-sized beetles by removing larvae from the carcass at various times after hatching (Steiger 2013; Pilakouta et al. 2015b, 2016b). Thus, for each of 100 broods, we removed third-instar larvae weighing $80-100,140-160$, and $200-220 \mathrm{mg}$ to generate small, medium-sized, and large adults, respectively. We kept each of these larvae in individual containers $(12 \mathrm{~cm} \times 8 \mathrm{~cm} \times$ $2 \mathrm{~cm}$ ) filled with moist soil until sexual maturity. After eclo- 
sion, we measured the pronotum length of all females to confirm that adults from the three groups differed in body size. As intended, there were clear differences in the mean ( $\pm \mathrm{SD}$ ) pronotum length $(\mathrm{mm})$ for the three groups: 2.89 ( \pm 0.14 ) for small females, 3.65 ( \pm 0.14 ) for mediumsized females, and $4.30( \pm 0.11)$ for large females.

We then set up contests over the possession of a carcass by pairing medium-sized females with either a small or large female competitor, which provided us with winners and losers of the same size. We expected the mediumsized female to win the contest if she was paired with a small female, whereas we expected her to lose the contest if she was paired with a large female. We only used females that had been sexually mature for up to 2 weeks (i.e., 10-24 days after eclosion), because female age has been shown to influence contest outcome in the closely related Nicrophorus orbicollis (Trumbo 2012). At the start of the experiment, we transferred pairs of females to transparent plastic containers $(17 \mathrm{~cm} \times 12 \mathrm{~cm} \times 6 \mathrm{~cm})$ with $1 \mathrm{~cm}$ of moist soil and a freshly thawed mouse carcass of a standardized size (20-22 g). We left the pairs undisturbed for 3 days, at which point we determined the winner by checking which beetle was present on the carcass (Safryn and Scott 2000; Trumbo 2012). In the vast majority of cases, the outcome of these contests was consistent with what we expected. However, when a medium-sized female won the contest to a large female or a medium-sized female lost the contest to a small female, she was excluded from the rest of the experiment. For the next part of the experiment, we allowed winners to breed on the mouse they had successfully competed for to mimic the outcome of winning a contest in the wild. In contrast, we prevented losers from breeding on the same mouse that was used for the contest, because losers would be driven away by the winner under natural conditions. Allowing losers to breed on the same mouse would have introduced uncertainty as to whether the female perceived herself to be the winner or loser of the contest. We therefore transferred losers to a new container $(17 \mathrm{~cm} \times 12 \mathrm{~cm} \times 6 \mathrm{~cm})$ with $1 \mathrm{~cm}$ of moist soil and a new mouse of the same size (20$22 \mathrm{~g})$. Winners and losers were mated with an unrelated virgin male immediately following the contest-that is, 3 days after they were paired up with a small or a large female, respectively.

In addition to the winner and loser treatments, we added two control treatments of medium-sized females that had no prior experience with a contest. Because losers had been exposed to two carcasses and winners had been exposed to only one, one of the control treatments matched the winner treatment, while the other control treatment matched the loser treatment. To this end, winner-control females were exposed to only one carcass, and a male was added to the box 3 days later. Meanwhile, loser-control females were ex- posed to two carcasses; they stayed on the first carcass for 3 days, at which time they were transferred to a different box with a new carcass of the same size and an unrelated virgin male. We also collected data on the females' prebreeding mass to be added as a covariate in our statistical models. For females exposed to only one carcass (winners and winner-controls), we measured prebreeding mass before placing the females on the carcass. For females exposed to two carcasses (losers and loser-controls), we measured prebreeding mass before placing the females on the second carcass, which was the one they bred on. The total sample size for this experiment was $n=224$ females, and the sample size for each treatment was as follows: $n=56$ for loser females, $n=57$ for loser-control females, $n=58$ for winner females, and $n=53$ for winner-control females.

To test for effects of contest outcome on prehatching reproductive effort, we recorded the number of eggs laid by each female and measured average egg size in each clutch. To do this, we checked the containers twice a day after mating for the presence of eggs. When the first eggs were laid, we removed the male to exclude any effects of male presence on the female's posthatching reproductive decisions. Immediately before hatching, we scanned the bottom of each container using a CanoScan 9000F Mark II scanner (Canon, Tokyo) to calculate egg size (Ford and Smiseth 2016). For each image, we measured the length and width of six randomly selected eggs in pixels using ImageJ (Abramoff et al. 2004; Monteith et al. 2012). We then converted these measurements to metric length $(\mathrm{mm})$ and calculated a prolate spheroid volume $V$ for each egg using the equation $V=(1 / 6) \pi w^{2} l$, where $w$ is the width and $l$ is the length of the egg (Berrigan 1991).

To test for effects of contest outcome on posthatching reproductive effort, we conducted behavioral observations for a random subset of broods $(n=15$ for loser females, $n=15$ for loser-control females, $n=17$ for winner females, and $n=18$ for winner-control females). This was done $24 \mathrm{~h}$ after the first larva hatched in each brood, as this stage in larval development corresponds to the peak in posthatching care in this species (Smiseth et al. 2003). We used instantaneous sampling every $1 \mathrm{~min}$ for $30 \mathrm{~min}$ in accordance with established protocols (Smiseth and Moore 2002; Mattey and Smiseth 2015b; Pilakouta et al. 2015b, 2016b). We recorded the number of scans that a female spent providing (i) direct care, defined as food provisioning to the larvae (i.e., mouth-tomouth contact with at least one larva) or interacting with the larvae (i.e., inside or around the crater and allowing larvae to beg), and (ii) indirect care, defined as carcass maintenance (i.e., deposition of secretions to the surface of the carcass or excavation of the crypt) or guarding (i.e., standing still in a position where it could defend the brood from predators or interspecific competitors). We also measured the larvae's begging rate as a proxy for offspring condition dur- 
ing early development (Smiseth and Moore 2004). To this end, we recorded the number of larvae begging at each scan, defined as larvae raising their head toward the parent while waving their legs or touching the parent with their legs (Smiseth and Moore 2002). From these data, we calculated an average begging rate for each brood, adjusting for brood size and the amount of time the parent spent in proximity to the larvae, given that larvae only beg in the presence of a parent (Rauter and Moore 1999; Smiseth and Moore 2002). This rate was based on the equation $b_{i}=\sum b / L \times 100 / p$, where $b_{i}$ is the percentage of time spent begging by each larva in the brood, $\sum b$ is the total number of larval begging events counted during the 30 scans of an observation session, $L$ is the brood size, and $p$ is the number of scans where the parent was in close proximity to the larvae (Smiseth and Moore 2002, 2004). At the end of the 30-min observation, we counted the number of larvae and weighed the whole brood. The larvae were returned to the carcass, and the female was allowed to care for the brood undisturbed until the larvae dispersed from the carcass about 4 days later.

At dispersal from the carcass, we recorded the size of the brood and total brood mass. We calculated average larval mass by dividing the total mass of the brood by the number of larvae in that brood. Brood size was used as a measure of the female's reproductive output. Last, females were transferred to individual containers filled with moist soil and were checked twice a week until death to record their postbreeding longevity, which is a measure of residual reproductive value (Boncoraglio and Kilner 2012).

\section{Data Analysis}

Raw data are available from the Dryad Digital Depository: http://dx.doi.org/10.5061/dryad.fq22f (Pilakouta et al. 2016a). All analyses were performed using $\mathrm{R}$, version 3.2.0. We used general linear models for traits with a normal distribution (egg size, larval begging rate, average larval mass at dispersal, and postbreeding longevity) and generalized linear models for traits with a Poisson distribution (amount of direct and indirect care) or a negative binomial distribution (egg number and brood size at dispersal). Correlations between all of our response variables are provided in table A1.

Our initial hypothesis was that the outcome of a prior contest would influence female reproductive decisions. Nevertheless, it was also possible that breeding females would adjust their reproductive investment simply due to the experience of a prior contest, regardless of its outcome. To disentangle potential effects due to experience with a contest and the outcome of that contest, all starting models included the following three factors: (i) prior contest experience, which compares the winners and losers to the controls (contest experience vs. no contest experience); (ii) number of mice encountered, which compares the winners and winner-controls to the losers and loser-controls (one mouse vs. two mice); and (iii) the interaction between these two factors, which reflects the effect of contest outcome (winning vs. losing). We also added female prebreeding mass and age at the time of mating as covariates in the models for egg number, egg size, brood size at dispersal, and larval mass at dispersal. The models for the behavioral data (direct care, indirect care, and larval begging rate) included carcass size, brood size, and average larval mass at the time of the observation as covariates, because parents might adjust the amount of care they provide based on the size of the carcass, the brood size, and the developmental stage of the larvae (Smiseth et al. 2003). Last, we included female age at the dispersal stage in the model for female postbreeding longevity. Decisions on whether to include any of the covariates in the final models were based on the lowest Akaike information criterion score.

\section{Results \\ Does Contest Experience or Outcome Influence Prehatching or Posthatching Effort?}

We found no evidence that prior experience with a contest or the outcome of that contest influenced prehatching in-

Table 1: Effects of contest experience (yes or no), the number of mice encountered by the female (one or two), and the interaction between these two factors (reflecting the effect of contest outcome) on female prehatching and posthatching investment

\begin{tabular}{|c|c|c|c|c|c|c|}
\hline & \multicolumn{2}{|c|}{ Contest experience } & \multicolumn{2}{|c|}{ No. mice } & \multicolumn{2}{|c|}{ Interaction } \\
\hline & LR $\chi_{1}^{2}$ & $P$ & $\operatorname{LR} \chi_{1}^{2}$ & $P$ & $\operatorname{LR} \chi_{1}^{2}$ & $P$ \\
\hline \multicolumn{7}{|l|}{ Prehatching investment: } \\
\hline Egg number & .05 & .82 & .41 & .52 & .47 & .50 \\
\hline Egg size & 1.06 & .30 & .01 & .92 & .28 & .60 \\
\hline \multicolumn{7}{|l|}{ Posthatching investment: } \\
\hline Amount direct care & 52.77 & $<.0001$ & .55 & .46 & .48 & .49 \\
\hline Amount indirect care & .42 & .52 & 3.21 & .07 & .29 & .59 \\
\hline
\end{tabular}

Note: $\mathrm{LR}=$ likelihood ratio. Statistically significant $P$ value $(<.05)$ is shown in boldface. 
vestment, as measured by egg number and egg size (tables 1 , A2). However, prior experience with a contest had a significant effect on posthatching investment, with winners and losers providing more direct care (food provisioning and interactions with the larvae) than control females (tables 1, A2; fig. 1A). There was no additional effect of the outcome of the contest on posthatching investment (see "Interaction" in table 1); winners and losers spent a similar amount of time providing direct care to the developing larvae (table A2; fig. 1A). There were no effects of either contest experience or contest outcome on the amount of time females spent providing indirect care (tables 1, A2). However, females provided more indirect care when breeding on smaller carcasses (likelihood ratio [LR] $\chi_{1}^{2}=6.25$, $P=.01)$ and when the larvae were larger at the time of
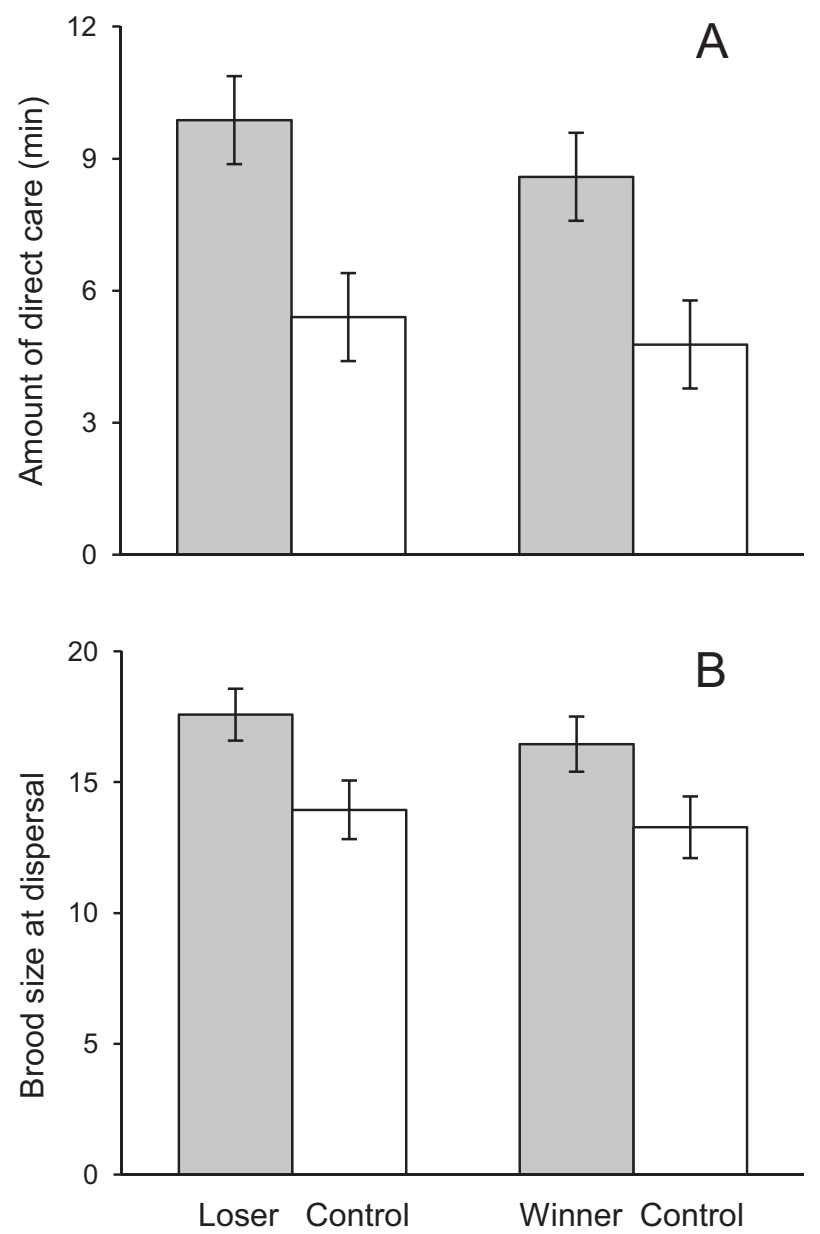

Figure 1: Amount of direct care $(A)$ and brood size at dispersal $(B)$ for females that either lost or won a prior contest and their corresponding controls. Error bars indicate SE. Losers and winners provided more direct care to their offspring $(A)$ and had larger broods $(B)$ than control females, which had no prior contest experience. There was no significant difference between losers and winners with respect to direct care $(A)$ or brood size at dispersal $(B)$. observation $\left(\operatorname{LR} \chi_{1}^{2}=21.0, P<.0001\right)$. There were no effects of the number of mice encountered on either prehatching or posthatching investment (table 1).

\section{Does Contest Experience or Outcome Have Fitness Consequences for Females or Their Offspring?}

Prior experience with a contest had a significant effect on the reproductive output of females, as winners and losers had larger broods at the dispersal stage than control females (table 2; fig. $1 B$ ). However, brood size was not influenced by the number of mice encountered by a female or the outcome of the contest (i.e., the interaction between number of mice and contest experience; table 2; fig. $1 B$ ). Female postbreeding longevity was not influenced by prior experience with a contest, the outcome of that contest, or the number of mice encountered by the female (tables 2, A2).

With respect to offspring fitness, none of the main factors had a significant effect on larval begging rate, but larger larvae spent more time begging than smaller larvae (LR $\left.\chi_{1}^{2}=11.2, P<.001\right)$, and there was a nonsignificant trend for larvae to beg more on larger carcasses (LR $\chi_{1}^{2}=$ 3.62, $P=.06$ ). Similarly, neither contest experience nor contest outcome had a detectable effect on average larval mass at dispersal (tables 2, A2; fig. 2A). However, females that had encountered two mice (losers and loser-controls) produced heavier offspring than females that encountered one mouse (winners and winner-controls). This pattern may have been driven by differences in prebreeding mass between these females (fig. $2 B$ ), given the correlation between the mother's prebreeding mass and the offspring's average larval mass (Pearson correlation: $r=0.31, P<$ .0001). Female prebreeding mass (LR $\chi_{1}^{2}=4.65, P=$ $.03)$ as well as female age $\left(\operatorname{LR} \chi_{1}^{2}=4.88, P=.03\right)$ were included in the final model for average larval mass. Because of the correlation between female prebreeding mass and number of mice encountered (fig. $2 B$ ), we tested for multicollinearity in this model. We estimated variance inflation factors using the vif function in the car package (Fox and Weisberg 2011). The largest variance inflation factors were $<2$, indicating absence of multicollinearity.

\section{Discussion}

Here, we tested the hypothesis that females increase their investment in current reproduction after losing a contest with a larger competitor and that such adjustments in investment alter the female's own fitness and the fitness of her offspring. We found no evidence to support this hypothesis, as contest outcome had no effect on either prehatching or posthatching investment (fig. 1A) and had no fitness consequences for either the female or her offspring (figs. $1 B, 2 A$ ). Instead, our key finding was that experience 
Table 2: Effects of contest experience (yes or no), the number of mice encountered by the female (one or two), and the interaction between these two factors (reflecting the effect of contest outcome) on the mother's reproductive output (brood size) and residual reproductive value (postbreeding longevity), offspring condition during early development (larval begging rate), and offspring size at the end of the parental care period (larval mass at dispersal)

\begin{tabular}{|c|c|c|c|c|c|c|}
\hline & \multicolumn{2}{|c|}{ Contest experience } & \multicolumn{2}{|c|}{ No. mice } & \multicolumn{2}{|c|}{ Interaction } \\
\hline & $\operatorname{LR} \chi_{1}^{2}$ & $P$ & $\overline{L R} \chi_{1}^{2}$ & $P$ & $\overline{L R} \chi_{1}^{2}$ & $\bar{P}$ \\
\hline \multicolumn{7}{|l|}{ Consequences for mother: } \\
\hline Brood size at dispersal & 8.01 & $<.01$ & $<.01$ & $>.99$ & .08 & .78 \\
\hline Postbreeding longevity & 1.34 & .25 & .49 & .48 & .01 & .91 \\
\hline \multicolumn{7}{|l|}{ Consequences for offspring: } \\
\hline Larval begging rate & .92 & .34 & 1.36 & .24 & .02 & .89 \\
\hline Larval mass at dispersal & .83 & .36 & 10.88 & $<.01$ & 2.27 & .13 \\
\hline
\end{tabular}

Note: LR $=$ likelihood ratio. Statistically significant $P$ values $(<.05)$ are shown in boldface.

with a prior contest, regardless of its outcome, influenced the subsequent reproductive decisions of females, thus altering their reproductive output. Both winners and losers spent almost twice as much time providing direct care (food provisioning and interactions with larvae) than females with no contest experience, and they had larger broods at the dispersal stage. Our findings are in line with two recent studies on winner-loser effects showing that contest experience might be more important than contest outcome in determining fighting success in future contests (Benelli et al. 2015a, 2015b).

One potential explanation for why both winners and losers increased their investment in the current brood is that females use the presence or absence of conspecific competitors as an indicator of the intensity of competition over limited breeding resources in the population. For example, if the absence of conspecific competitors indicates a low population density, control females may have invested less in their current brood to take advantage of additional breeding opportunities in the future (McNamara et al. 2009). An alternative explanation is that involvement in a contest might have resulted in injuries given the fierce competition over carcasses in Nicrophorus (Trumbo 1991; Cotter et al. 2011), and injured females might have increased their investment in the current brood due to a higher risk of infection. A previous study on Nicrophorus vespilloides found that immunologically challenged females produced heavier broods than control females (Cotter et al. 2011). Nevertheless, we observed visible injuries in the form of missing antennae or legs only in one loser, and we never observed such injuries in any of the winners. Thus, the most likely explanation for the observation that winners and losers increased their investment in current reproduction is that they responded to the presence of a competitor, which served as a cue about the intensity of competition in the population.

As a result of this increased investment, winners and losers produced more offspring at the dispersal stage than control females. To our knowledge, this is the first evidence that a female's prior experience with a contest influences her reproductive output by altering her subsequent parental investment decisions. On the other hand, we found no evidence for an effect of either contest experience or contest outcome on the offspring's fitness. This result was somewhat surprising given that larval mass determines adult size, which in turn determines the likelihood of acquiring a carcass for breeding (Bartlett and Ashworth 1988; Safryn and Scott 2000; Lock et al. 2004). Thus, we might have expected an adjustment in offspring size by females that had prior contest experience, due to an anticipatory maternal response to the intense levels of competition at high population density (Creighton 2005). Instead, we found that offspring size depended on the number of mice females had encountered. Losers and loser-controls, which had encountered two mice, produced larger larvae compared to winners and winner-controls, which had encountered only one mouse. This pattern might be driven by the higher mass of losers and loser-controls (fig. $2 B$ ), which had access to an additional carcass before being placed on the mouse they eventually bred on. Mouse carcasses are a highly nutritional food resource for burying beetles, and parents feed on the carcass before and during a breeding attempt to replenish their energy reserves (Pilakouta et al. 2016b). If losers and losercontrols had more energy reserves at the start of their breeding attempt, they might have consumed less of the second carcass, leaving more food for the larvae.

Overall, our results show that females with prior contest experience invest more in current reproduction and produce larger broods, but they do not adjust their offspring's size. This finding is in contrast to studies in other species showing that mothers produce fewer but larger offspring at high densities (Both 2000; Creighton 2005; Goubault et al. 2007; Plaistow et al. 2007; Allen et al. 2008; Leips et al. 2009). Thus, there is mixed empirical evidence with respect to how females adjust the number versus the size of their offspring in response to intense levels of competition. This highlights the 

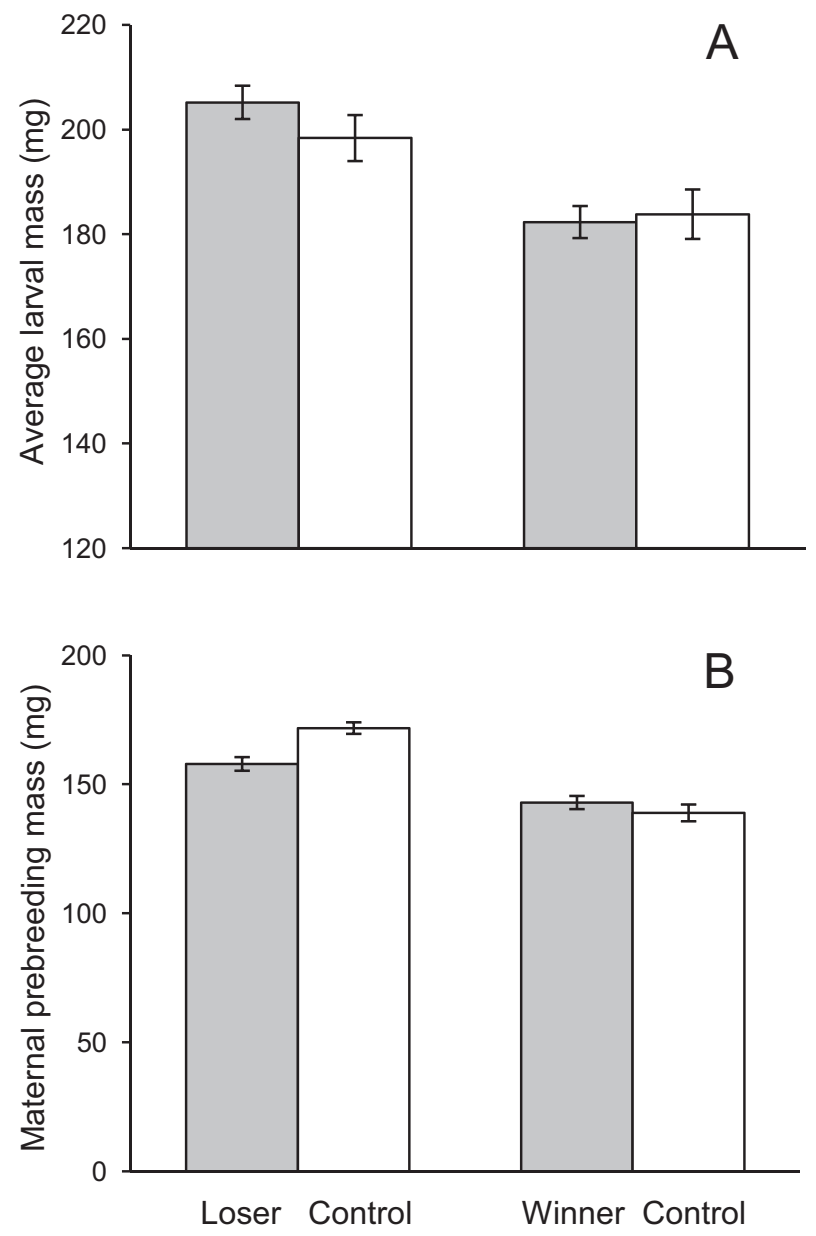

Figure 2: Average larval mass $(A)$ and maternal prebreeding mass $(B)$ for females that either lost or won a prior contest and their corresponding controls. Error bars indicate SE. For females exposed to only one carcass (winners and winner-controls), we measured prebreeding mass before placing the females on the carcass. For females exposed to two carcasses (losers and loser-controls), we measured prebreeding mass before placing the females on the second carcass, which was the one they bred on.

need for further work on this topic, given that different scenarios for how females respond to high population density could have divergent consequences for population dynamics.

To illustrate this issue, we propose a conceptual model based on the simple assumption that variation in population density determines the intensity of intraspecific competition over resources used for breeding (Creighton 2005). When population density is low, there will be little competition over resources. Under these conditions, most breeding individuals will have no experience with a prior contest, in which case they may show reproductive restraint because they have a relatively high reproductive potential. Conversely, when population density is high, the majority of breeding individuals will encounter competitors, leading to potential adjustments in their subsequent reproductive decisions. If females produce more offspring of the same size by increasing their investment in current reproduction, a greater number of offspring will be recruited into the breeding population at high densities. On the other hand, if females produce fewer but larger offspring without increasing their overall investment in the current brood, a smaller number of offspring will be recruited into the breeding population at high densities. Given that offspring recruitment into the breeding population is inextricably linked to population growth, these interactions could create a feedback loop between population density, intraspecific competition, investment in current reproduction, and offspring recruitment into the population.

Such feedback loops could occur in any species where there is size-dependent competition over resources and parents make reproductive investment decisions based on cues about the population density. Evidence for densitydependent adjustments in the number and/or size of offspring has been documented in a variety of taxa, including birds, fishes, insects, and aquatic invertebrates (Both 2000; Creighton 2005; Goubault et al. 2007; Plaistow et al. 2007; Allen et al. 2008; Leips et al. 2009; Rauter et al. 2010). In most of these studies, parents produced fewer, larger offspring at high densities and more, smaller offspring at low densities. Under this scenario, we would expect a negative feedback loop, where the population density fluctuates around a stable equilibrium. In contrast, if parents produce more offspring (of the same size) at high densities, as we found here, this would lead to a positive feedback loop. These two scenarios focus on how parents might adjust their investment during the current breeding attempt, but these adjustments might also have implications for future reproduction, which will in turn contribute to population dynamics. Parents that increase their overall investment in current reproduction are expected to suffer future costs in the form of a smaller second brood (Creighton et al. 2009; Ward et al. 2009; Billman et al. 2014), whereas there might not be any future costs associated with merely adjusting the trade-off between number and size of offspring. Theoretical modeling and long-term field studies are now needed to better understand these dynamics. A suitable data set would provide multigeneration information on parental investment patterns (e.g., incubation time or nestling provisioning rate for birds), clutch size, offspring size, offspring recruitment into the population, and estimated population size.

In summary, we propose that in species where contest experience mediates parental adjustments in the size and/ or number of offspring, the frequency of intraspecific contests could both influence and be influenced by population dynamics. This potential link between individual-level behavioral changes and population-level processes has so far been overlooked in the literature. More generally, previous stud- 
ies have largely ignored the wider fitness consequences of contest experience and contest outcome beyond an effect on success in subsequent fights. Our finding that contest experience can alter a female's reproductive decisions highlights the need for further empirical work in this area. Even though we did not find evidence for an effect of contest outcome on reproductive investment in this study, such an effect may still exist in other systems, making this an interesting question for future studies to explore. Last, although our study focused on parental investment, participation in a fighting contest might influence a variety of other behaviors and life-history trade-offs, and we encourage future research to consider these effects.

\section{Conclusion}

This study provides the first evidence that a female's experience with a prior contest can have consequences for her reproductive output through an adjustment in parental care. Winners and losers spent more time providing care to their offspring than females with no contest experience, resulting in larger broods at the end of the parental care period. In species where parents adjust their reproductive strategies based on their contest experience and where these adjustments affect the number and/or size of their offspring, contests could influence and be influenced by population dynamics, leading to a feedback loop between local-scale social interactions, individual-level behavioral changes, and population-level processes.

\section{Acknowledgments}

We thank the Edinburgh Countryside Rangers for permission to collect beetles at Craiglockhart Hill and D. Rozen for supplying beetles from the Netherlands. We also thank L. Kruuk, J. Moorad, and M. Paquet for helpful comments on the manuscript and statistical analysis. The study was funded by Campus Hungary (R.R.) and the Institute of Evolutionary Biology and School of Biological Sciences at the University of Edinburgh (N.P./C.H./P.T.S.).

\section{APPENDIX}

\section{Supplementary Tables}

Table A1: Correlation matrix for all response variables

\begin{tabular}{|c|c|c|c|c|c|c|c|}
\hline & Egg number & Egg size & Direct care & Indirect care & $\begin{array}{c}\text { Larval } \\
\text { begging rate }\end{array}$ & Brood size & $\begin{array}{c}\text { Average } \\
\text { larval mass }\end{array}$ \\
\hline Egg size & $\begin{aligned} r & =.15 \\
P & =.12\end{aligned}$ & & & & & & \\
\hline Direct care & $\begin{aligned} r & =.03 \\
P & =.92\end{aligned}$ & $\begin{aligned} r & =-.40 \\
P & =.20\end{aligned}$ & & & & & \\
\hline Indirect care & $\begin{aligned} r & =.82 \\
\boldsymbol{P} & =.001\end{aligned}$ & $\begin{aligned} r & =.29 \\
P & =.36\end{aligned}$ & $\begin{aligned} r & =.05 \\
P & =.66\end{aligned}$ & & & & \\
\hline Larval begging rate & $\begin{aligned} r & =.60 \\
P & =.40\end{aligned}$ & $\begin{array}{l}r=.08 \\
P=.93\end{array}$ & $\begin{aligned} r & =-.28 \\
P & =.07\end{aligned}$ & $\begin{aligned} r & =.04 \\
P & =.82\end{aligned}$ & & & \\
\hline Brood size & $\begin{array}{l}r=.75 \\
P<.001\end{array}$ & $\begin{array}{l}r=.36 \\
P<.001\end{array}$ & $\begin{aligned} r & =.20 \\
P & =.11\end{aligned}$ & $\begin{aligned} r & =.13 \\
P & =.32\end{aligned}$ & $\begin{aligned} r & =-.03 \\
P & =.87\end{aligned}$ & & \\
\hline Average larval mass & $\begin{aligned} r & =-.24 \\
P & =.01\end{aligned}$ & $\begin{aligned} r & =.29 \\
P & =.05\end{aligned}$ & $\begin{aligned} r & =.27 \\
P & =.03\end{aligned}$ & $\begin{aligned} r & =.19 \\
P & =.15\end{aligned}$ & $\begin{aligned} r & =-.05 \\
P & =.78\end{aligned}$ & $\begin{aligned} r & =-.14 \\
P & =.06\end{aligned}$ & \\
\hline Postbreeding longevity & $\begin{aligned} r & =.212 \\
P & =.03\end{aligned}$ & $\begin{aligned} r & =.24 \\
P & =.01\end{aligned}$ & $\begin{aligned} r & =-.09 \\
P & =.53\end{aligned}$ & $\begin{aligned} r & =.22 \\
P & =.45\end{aligned}$ & $\begin{aligned} r & =.27 \\
P & =.16\end{aligned}$ & $\begin{aligned} r & =.25 \\
\boldsymbol{P} & =.001\end{aligned}$ & $\begin{aligned} r & =.02 \\
P & =.81\end{aligned}$ \\
\hline
\end{tabular}

Note: Statistically significant $P$ values are shown in boldface (based on $\alpha=0.002$ due to Bonferroni correction).

Table A2: Means ( \pm SE) for female prehatching investment (egg number and egg size), posthatching investment (amount of direct and indirect care), maternal reproductive output (brood size), maternal residual reproductive value (postbreeding longevity), and fitness-related offspring traits (larval begging rate and larval mass at dispersal)

\begin{tabular}{lcccc}
\hline & Loser & Loser-control & Winner & Winner-control \\
\hline Prehatching investment: & & & & \\
$\quad$ Egg number & $17.3 \pm 1.7$ & $18.3 \pm 1.5$ & $19.1 \pm 1.7$ & $17.6 \pm 1.9$ \\
$\quad$ Egg size $\left(\mathrm{mm}^{3}\right)$ & $1.47 \pm .03$ & $1.42 \pm .03$ & $1.44 \pm .03$ & $1.43 \pm .03$ \\
$\quad \begin{array}{l}\text { Posthatching investment: } \\
\quad \text { Amount direct care (min) }\end{array}$ & $9.88 \pm 1.90$ & $5.40 \pm 1.99$ & $8.59 \pm 1.79$ & $4.78 \pm 1.21$
\end{tabular}


Table A2 (Continued)

\begin{tabular}{lcccc}
\hline & Loser & Loser-control & Winner & Winner-control \\
\hline $\begin{array}{l}\text { Amount indirect care (min) } \\
\text { Consequences for mother: }\end{array}$ & $4.27 \pm 1.01$ & $4.67 \pm 1.08$ & $5.41 \pm 1.05$ & $4.78 \pm .93$ \\
$\quad$ & $17.6 \pm 1.0$ & $13.9 \pm 1.1$ & $16.4 \pm 1.1$ & $13.3 \pm 1.2$ \\
$\quad$ Brood size at dispersal & $44.6 \pm 2.0$ & $42.1 \pm 2.0$ & $42.8 \pm 2.1$ & $40.8 \pm 1.9$ \\
$\quad \begin{array}{l}\text { Postbreeding longevity (days) } \\
\text { Consequences for offspring: }\end{array}$ & $15.7 \pm 5.1$ & $22.7 \pm 9.3$ & $9.7 \pm 3.0$ & $14.7 \pm 4.3$ \\
$\quad$ Larval begging rate (\%) & $205 \pm 3$ & $198 \pm 4$ & $182 \pm 3$ & $184 \pm 5$ \\
$\quad$ Larval mass at dispersal (mg) & & &
\end{tabular}

\section{Literature Cited}

Abramoff, M. D., P. J. Magalhaes, and S. J. Ram. 2004. Image processing with ImageJ. Biophotonics International 11:36-42.

Allen, R. M., Y. M. Buckley, and D. J. Marshall. 2008. Offspring size plasticity in response to intraspecific competition: an adaptive maternal effect across life stages. American Naturalist 171:225-237.

Bartlett, J., and C. M. Ashworth. 1988. Brood size and fitness in Nicrophorus vespilloides (Coleoptera: Silphidae). Behavioral Ecology and Sociobiology 22:429-434. doi:10.1007/BF00294981.

Benelli, G., N. Desneux, D. Romano, G. Conte, R. H. Messing, and A. Canale. 2015a. Contest experience enhances aggressive behaviour in a fly: when losers learn to win. Scientific Reports 5:9347. doi:10 $.1038 /$ srep09347.

Benelli, G., D. Romano, N. Desneux, R. H. Messing, and A. Canale. 2015b. Sex differences in fighting-induced hyperaggression in a fly. Animal Behaviour 104:165-174. doi:10.1016/j.anbehav.2015.02.026.

Berrigan, D. 1991. The allometry of egg size and number in insects. Oikos 60:313-321. doi:10.2307/3545073.

Billman, E. J., J. C. Creighton, and M. C. Belk. 2014. Prior experience affects allocation to current reproduction in a burying beetle. $\underline{\text { Be- }}$ havioral Ecology 25:813-818. doi:10.1093/beheco/aru051.

Boncoraglio, G., and R. M. Kilner. 2012. Female burying beetles benefit from male desertion: sexual conflict and counter-adaptation over parental investment. PLoS ONE 7:e31713. doi:10.1371/journal .pone.0031713.

Both, C. 2000. Density dependence of avian clutch size in resident and migrant species: is there a constraint on the predictability of competitor density? Journal of Avian Biology 31:412-417.

Chase, I., C. Bartolomeo, and L. Dugatkin. 1994. Aggressive interactions and inter-contest interval: how long do winners keep winning? Animal Behaviour 48:393-400. doi:10.1006/anbe.1994.1253.

Cotter, S. C., R. J. S. Ward, and R. M. Kilner. 2011. Age-specific reproductive investment in female burying beetles: independent effects of state and risk of death. Functional Ecology 25:652-660. doi:10.1111/j.1365-2435.2010.01819.x.

Creighton, J. C. 2005. Population density, body size, and phenotypic plasticity of brood size in a burying beetle. Behavioral Ecology 16 1031-1036. doi:10.1093/beheco/ari084.

Creighton, J. C., N. D. Heflin, and M. C. Belk. 2009. Cost of reproduction, resource quality, and terminal investment in a burying beetle. American Naturalist 174:673-684. doi:10.1086/605963.

Eggert, A.-K., M. Reinking, and J. K. Müller. 1998. Parental care improves offspring survival and growth in burying beetles. Animal Behaviour 55:97-107. doi:10.1006/anbe.1997.0588.

Fawcett, T. W., and R. A. Johnstone. 2010. Learning your own strength: winner and loser effects should change with age and experience. Proceedings of the Roval Societv B: Biological Sciences 277:1427-1434
Fetherston, I. A., M. P. Scott, and J. F. A. Traniello. 1994. Behavioural compensation for mate loss in the burying beetle Nicrophorus orbicollis. Animal Behaviour 47:777-785. doi:10.1006/anbe.1994.1110.

Ford, L. E., and P. T. Smiseth. 2016. Asynchronous hatching provides females with a means for increasing male care but incurs a cost by reducing offspring fitness. Journal of Evolutionary Biology 29:428-437.

Fox, J., and S. Weisber. 2011. An R companion to applied regression. 2nd ed. Sage, Thousand Oaks, CA. http://socserv.socsci.mcmaster.ca/jfox /Books/Companion.

Goubault, M., A. F. S. Mack, and I. C. W. Hardy. 2007. Encountering competitors reduces clutch size and increases offspring size in a parasitoid with female-female fighting. Proceedings of the Roval Societv B: Biological Sciences 274:2571-2577.

Hsu, Y., R. Earley, and L. L. Wolf. 2006. Modulation of aggressive behaviour by fighting experience: mechanisms and contest outcomes. Biological Reviews 81:33-74. doi:10.1017/S146479310500686X.

Hsu, Y., and L. L. Wolf. 2001. The winner and loser effect: what fighting behaviours are influenced? Animal Behaviour 61:777-786. doi:0 $.1006 /$ anbe.2000.1650.

Kasumovic, M. M., D. O. Elias, S. Sivalinghem, A. C. Mason, and M. C. B. Andrade. 2010. Examination of prior contest experience and the retention of winner and loser effects. Behavioral Ecology 21: 404-409. doi:10.1093/beheco/arp204.

Leips, J., J. M. L. Richardson, F. H. Rodd, and J. Travis. 2009. Adaptive maternal adjustments of offspring size in response to conspecific density in two populations of the least killifish, Heterandria formosa. Evolution 63:1341-1347.

Lock, J. E., P. T. Smiseth, and A. J. Moore. 2004. Selection, inheritance, and the evolution of parent-offspring interactions. American Naturalist 164:13-24. doi:10.1086/421444.

Mattey, S. N., and P. T. Smiseth. 2015a. No inbreeding avoidance by female burying beetles regardless of whether they encounter males simultaneously or sequentially. Ethology 121:1031-1038. doi:10.1111 /eth.12417.

- 2015b. Complex effects of inbreeding on biparental cooperation. American Naturalist 185:1-12. doi:10.1086/679067.

McNamara, J. M., A. I. Houston, Z. Barta, A. Scheuerlein, and L. Fromhage. 2009. Deterioration, death and the evolution of reproductive restraint in late life. Proceedings of the Roval Society B: Biological Sciences 276:4061-4066. doi:10.1098/rspb.2009.0959.

Monteith, K. M., C. Andrews, and P. T. Smiseth. 2012. Post-hatching parental care masks the effects of egg size on offspring fitness: a removal experiment on burying beetles. Journal of Evolutionary Biology 25:1815-1822. doi:10.1111/j.1420-9101.2012.02567.x.

Okada, K., T. Yamane, and T. Miyatake. 2010. Ejaculatory strategies associated with experience of losing. Biology Letters 6:593-596. doi:10.1098/rsbl.2010.0225. 
Otronen, M. 1990. The effect of prior experience on the outcome of fights in the burying beetle, Nicrophorus humator. Animal Behaviour 40:980-982. doi:10.1016/S0003-3472(05)81000-0.

Pilakouta, N., C. Halford, R. Rácz, and P. T. Smiseth. 2016a. Data from: Effects of prior contest experience and contest outcome on female reproductive decisions and offspring fitness. American Naturalist, Dryad Digital Repository, http://dx.doi.org/10.5061/dryad.fq22f.

Pilakouta, N., S. Jamieson, J. A. Moorad, and P. T. Smiseth. $2015 a$. Parental care buffers against inbreeding depression in burying beetles. Proceedings of the National Academy of Sciences of the USA 112 8031-8035. doi:10.1073/pnas.1500658112.

Pilakouta, N., J. Richardson, and P. T. Smiseth. 2015b. State-dependent cooperation in burying beetles: parents adjust their contribution towards care based on both their own and their partner's size. Journal of Evolutionary Biology 28:1965-1974. doi:10.1111/jeb.12712.

. 2016b. If you eat, I eat: resolution of sexual conflict over feeding from a shared resource. Animal Behaviour 111:175-180. doi:10 .1016/j.anbehav.2015.10.016.

Plaistow, S. J., J. H. H. St. Clair, J. Grant, and T. G. Benton. 2007. How to put all your eggs in one basket: empirical patterns of offspring provisioning throughout a mother's lifetime. American Naturalist 170:520-529.

Rauter, C. M., M. J. McGuire, M. M. Gwartney, and J. E. Space. 2010 Effect of population density and female body size on number and size of offspring in a species with size-dependent contests over resources. Ethology 116:120-128.

Rauter, C. M., and A. J. Moore. 1999. Do honest signalling models of offspring solicitation apply to insects? Proceedings of the Royal Society B: Biological Sciences 266:1691-1696.

- 2004. Time constraints and trade-offs among parental care behaviours: effects of brood size, sex and loss of mate. Animal Behaviour 68:695-702. doi:10.1016/j.anbehav.2003.09.018.

Rozen, D. E., D. J. P. Englemoer, and P. T. Smiseth. 2008. Antimicrobial strategies in burying beetles breeding on carrion. Proceedings of the National Academy of Sciences of the USA 105:1789017895.

Rutte, C., M. Taborsky, and M. Brinkhof. 2006. What sets the odds of winning and losing? Trends in Ecology and Evolution 21:16-21. doi:10.1016/j.tree.2005.10.014
Safryn, S., and M. Scott. 2000 Sizing up the competition: do burying beetles weigh or measure their opponents? Journal of Insect Behavior 13:291-297. doi:10.1023/A:1007700601095.

Smiseth, P. T., C. Darwell, and A. J. Moore. 2003. Partial begging: an empirical model for the early evolution of offspring signalling. Proceedings of the Royal Society B: Biological Sciences 270:17731777. doi:10.1098/rspb.2003.2444.

Smiseth, P. T., C. Dawson, E. Varley, and A. J. Moore. 2005. How do caring parents respond to mate loss? differential response by males and females. Animal Behaviour 69:551-559. doi:10.1016/j.anbehav .2004.06.004.

Smiseth, P. T., and A. J. Moore. 2002. Does resource availability affect offspring begging and parental provisioning in a partially begging species? Animal Behaviour 63:577-585. doi:10.1006/anbe.2001 .1944.

. 2004. Signalling of hunger when offspring forage by both begging and self-feeding. Animal Behaviour 67:1083-1088.

Steiger, S. 2013. Bigger mother are better mothers: disentangling sizerelated prenatal and postnatal maternal effects. Proceedings of the Royal Society B: Biological Sciences 280:20131225. doi:10.1098/rspb .2013.1225.

Trumbo, S. T. 1991. Reproductive benefits and the duration of paternal care in a biparental burying beetle, Necrophorus orbicollis. Behaviour 117:82-105

- 2012. Contest behavior and other reproductive efforts in aging breeders: a test of residual reproductive value and statedependent models. Behavioral Ecology and Sociobiology 66:15111518. doi:10.1007/s00265-012-1406-4.

Walling, C. A., C. E. Stamper, C. L. Salisbury, and A. J. Moore. 2008 Experience does not alter alternative mating tactics in the burying beetle Nicrophorus vespilloides. Behavioral Ecology 20:153-159. doi:10.1093/beheco/arn127.

Ward, R. J. S., S. C. Cotter, and R. M. Kilner. 2009. Current brood size and residual reproductive value predict offspring desertion in the burying beetle Nicrophorus vespilloides. Behavioral Ecology 20:1274-1281. doi:10.1093/beheco/arn132.

Associate Editor: Roger K. Butlin Editor: Judith L. Bronstein

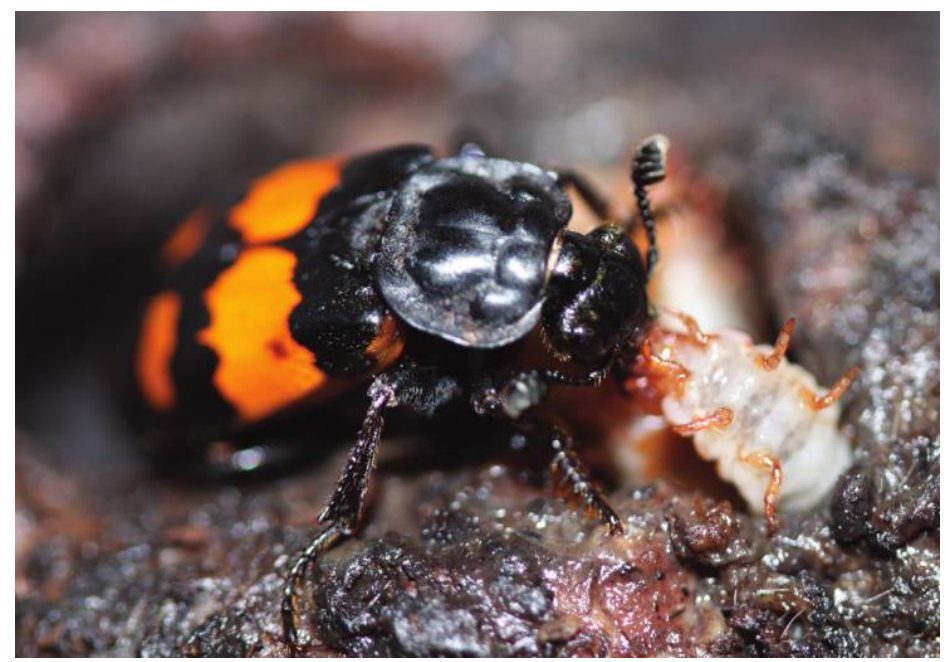

A female burying beetle (Nicrophorus vespilloides) feeding predigested carrion to a begging larva. Photo credit: Per T. Smiseth. 